\title{
Flow and geometry induced scattering of high frequency acoustic duct modes
}

\author{
A.F. Smith, N.C. Ovenden*, R.I. Bowles \\ Department of Mathematics, University College London, Gower St, London WC1E 6BT, United Kingdom
}

\section{A R T I C L E I N F O}

\section{Article history:}

Received 4 February 2011

Received in revised form 24 June 2011

Accepted 27 July 2011

Available online 9 August 2011

\section{Keywords:}

Duct modes

Mean flow

Scattering

Standing waves

\begin{abstract}
A B S T R A C T
Cut-on cut-off transition of acoustic modes in hard-walled ducts with irrotational mean flow is well understood for Helmholtz numbers of order unity. Previous finite-element simulations of this phenomenon, however, appear to indicate the possibility of energy scattering into neighbouring modes at moderately large Helmholtz numbers. In this paper, such scattering phenomena are explained and predicted in slowly varying aeroengine ducts using a multiple-scales approach. It is found that, for sufficiently high frequencies, two mechanisms exist whereby energy can be scattered into neighbouring modes by an incident propagating mode. One mechanism occurs only when there is a mean flow inside the duct and induces scattering at significantly lower frequencies than the other mechanism which remains present without mean flow. A coupled system of ordinary differential equations is derived and then solved numerically for a number of example cases to obtain the corresponding transmitted and reflected amplitudes of the scattered modes as well as the overall acoustic pressure field. The theory appears to demonstrate that some exchange of energy between the acoustic and mean flow fields occurs during scattering.
\end{abstract}

(C) 2011 Elsevier B.V. All rights reserved.

\section{Introduction}

The propagation of unsteady disturbances in ducts of slowly-varying geometry, such as those typical of an aeroengine, can be successfully modelled using a multiple scales approach. From the first application [1] of multiple-scales analysis to sound propagation in ducts of rectangular and circular cross section without mean flow, more recent developments have extended the method to cases with uniform mean flow [2], mean swirling flow [3], ducts of arbitrary cross section [4] (with uniform mean flow) and strongly curved ducts [5]. The multiple-scales approach has a number of distinct advantages over full numerical methods as it is ideally suited to handle higher frequencies and the computational complexity is only marginally more than calculating the eigenmodes inside a straight parallel duct. The accuracy and usefulness of the multiple scales approach has been validated against finite-element methods [6] for realistic aeroengine configurations and acoustic frequencies $[7,8]$.

Cut-on cut-off transition of acoustic modes in hard-walled ducts with irrotational mean flow is well understood, where an analysis within an inner scaling region around the singularity in the multiple-scales solution for the incident mode shows that the mode undergoes a total reflection with a phase shift of $\pi / 2$ [9-11]. Significantly for typical aeroengine applications, a similar partial reflection also occurs in lined ducts of finite impedance [12]. However, a recent comparison paper [8] contains finite-element simulations of cut-on cut-off transition that appear to indicate the possibility of energy scattering into neighbouring modes at large Helmholtz numbers. Out of the seven benchmark cases presented in that comparison paper, modal scattering was observed in the high-frequency cases with a high cross-sectional eigenvalue for the incident

\footnotetext{
* Corresponding author.

E-mail address: nicko@math.ucl.ac.uk (N.C. Ovenden).
} 
mode, and where a mean-flow is present within the duct. A brief argument put forward by the authors of the paper was based on the smaller separation of neighbouring eigenvalues at high frequency and it was conjectured that scattering may occur at non-dimensional frequencies of the order of $\epsilon^{-2}$, where $\epsilon \ll 1$ is the so-called slowly-varying parameter (a typical feature of the nondimensional duct geometry variation as described below).

In this paper, we attempt to explain and predict such observed scattering phenomena in slowly varying aeroengine ducts using a multiple-scales approach. Our analysis appears to show firstly that the order of magnitude estimate obtained previously [8] of the nondimensional frequency required for modal scattering to occur appears overestimated when a mean flow is present within the duct. Furthermore we show that, for sufficiently high frequencies, two mechanisms exist whereby energy can be scattered into neighbouring modes by an incident propagating mode undergoing cut-on cut-off transition. The first mechanism is described in Section 4 and is induced by the mean flow inside the duct; it is significant for Helmholtz numbers of only $O\left(\epsilon^{-1 / 2}\right)$ or larger. Indeed, we demonstrate that this flow-induced scattering mechanism leads to direct coupling and some exchange of energy with the mean flow. The second geometry-induced scattering mechanism is derived in Section 5 and generates significant scattering only at much larger Helmholtz numbers of $O\left(\epsilon^{-2}\right)$ or higher, more in line with the previous prediction. Examples of scattering via both mechanisms are then presented for a duct of rectangular cross-section. The straightforward application of the theory to ducts of both circular and annular cross-section is described towards the end of the paper.

\section{Governing equations}

We begin by considering a compressible inviscid isentropic irrotational gas flow inside a duct of slowly-varying cross section. The ratio of specific heats at constant pressure and volume is taken to be $\gamma$ and it is assumed that (i) the acoustic oscillations are too rapid for heat conduction and (ii) the viscous forces are also assumed to be negligible. The governing Euler equations and perfect gas relation are made dimensionless by scaling all spatial dimensions on a typical duct width or diameter $h_{0}$, the gas density $\tilde{\rho}$ on an ambient reference value $\rho_{0}$, the velocity field $\tilde{\mathbf{v}}$ and sound speed $\tilde{c}$ on an ambient reference sound speed $c_{0}$, time on $h_{0} / c_{0}$ and pressure $\tilde{p}$ on $\rho_{0} c_{0}^{2}$. The flow field can subsequently be expressed as the sum of a mean flow plus infinitesimally small time-harmonic perturbations of non-dimensional frequency (Helmholtz number) $\omega$,

$$
[\tilde{\mathbf{v}}, \tilde{\rho}, \tilde{p}, \tilde{c}]=[\mathbf{V}, D, P, C]+[\nabla \phi, \rho, p, c] \mathrm{e}^{\mathrm{i} \omega t} .
$$

The duct geometry is described using Cartesian coordinates $(x, y, z)$, with corresponding unit vectors $\mathbf{e}_{\mathbf{x}}, \mathbf{e}_{\mathbf{y}}, \mathbf{e}_{\mathbf{z}}$, so that the duct cross section lies in the $y z$-plane and varies slowly in the axial $x$-direction. For ducts of cylindrical or annular crosssection, cylindrical coordinates are used $(x, r, \theta)$, where $(y, z)=(r \cos \theta, r \sin \theta)$. Defining $X=\epsilon X$ (with $\epsilon \ll 1)$ as a slow axial scale, the mean flow is assumed to take the form

$$
\begin{aligned}
& \mathbf{V}(X, y, z ; \epsilon)=U_{0}(X) \mathbf{e}_{\mathbf{x}}+\epsilon \mathbf{V}_{\perp}(X, y, z)+O\left(\epsilon^{2}\right), \\
& {[D, C, P]=\left[D_{0}(X), C_{0}(X), P_{0}(X)\right]+O\left(\epsilon^{2}\right),}
\end{aligned}
$$

where $\mathbf{V}_{\perp}(X, y, z)$ represents the crosswise mean flow component. The leading order solution to the mean flow expansions can be determined [4], given the cross-sectional area $A(X)$ and two constant flow parameters $\mathscr{E}$ and $\mathcal{F}$, by solving

$$
U_{0}(X)=\frac{\mathcal{F}}{D(X) A(X)}, \quad \text { and } \quad \frac{\mathcal{F}^{2}}{2 D^{2}(X) A^{2}(X)}+\frac{D^{\gamma-1}(X)}{\gamma-1}=\varepsilon .
$$

The crosswise mean velocity is subsequently determined by mass conservation,

$$
\frac{\partial}{\partial X}\left(U_{0} D_{0}\right)+\nabla_{\perp} \cdot\left(D_{0} \mathbf{V}_{\perp}\right)=0 .
$$

For the acoustic perturbations, the acoustic velocity potential $\phi$ satisfies the general convective wave equation [13], given by

$$
\nabla \cdot(D \nabla \phi)-D(\mathrm{i} \omega+\mathbf{V} \cdot \nabla)\left[C^{-2}(\mathrm{i} \omega+\mathbf{V} \cdot \nabla) \phi\right]=0 .
$$

On obtaining a solution to $\phi$, the acoustic pressure $p$ and density perturbation $\rho$ can be recovered from $\phi$ via the relations

$$
p=-D(\mathrm{i} \omega+\mathbf{V} \cdot \nabla) \phi, \text { and } p=C^{2} \rho .
$$

\section{The multiple-scales solution and turning points}

For $\omega \sim 1$, substituting the mean flow solution from Eq. (2) into the convective wave equation (4) yields

$$
\frac{\partial^{2} \phi}{\partial x^{2}}+\nabla_{\perp}^{2} \phi-\frac{1}{C_{0}^{2}}\left[-\omega^{2} \phi+2 i \omega U_{0} \frac{\partial \phi}{\partial x}+U_{0}^{2} \frac{\partial^{2} \phi}{\partial x^{2}}\right]=O(\epsilon),
$$

where $\nabla_{\perp}=\left(0, \partial_{y}, \partial_{z}\right)$ and the right-hand side of (6) contains the so-called non-parallel terms due to the mean flow variation. The solution to this equation can be expressed to leading-order as a sum of slowly-varying modes with given incident amplitudes $Q_{k}$ as follows $[2,4,9,11]$ 


$$
\phi=\sum_{k=-\infty}^{\infty} Q_{k} \sqrt{\frac{C_{0}(X)}{\omega D_{0}(X) \sigma_{k}(X)}} \psi_{|k|}(y, z ; X) \exp \left(-\frac{\mathrm{i}}{\epsilon} \int^{X} \frac{\omega\left(C_{0} \sigma_{k}-U_{0}\right)}{\left(C_{0}^{2}-U_{0}^{2}\right)} \mathrm{d} X^{\prime}\right) .
$$

Here, the function $\psi_{|k|}$ solves the slowly-varying cross-sectional eigenvalue problem $\nabla_{\perp}^{2} \psi_{|k|}=-\alpha_{|k|}^{2}(X) \psi_{|k|}$, for eigenvalues $\alpha_{|k|}(X)$, with boundary conditions that $\nabla \psi_{|k|} \cdot \mathbf{n}=0$ at the duct walls with normal vector $\mathbf{n}$. It is normalised, as in [4], by integrating over the duct cross section, a domain given by $\mathscr{A}(X)$, and imposing $\iint_{\mathscr{A}(X)} \psi_{|k|}^{2} \mathrm{~d} y \mathrm{~d} z=1$. The slowly varying term $\sigma_{k}(X)$ in the expression is the so-called reduced axial wavenumber [2] and it is given by

$$
\sigma_{k}(X)=\operatorname{sgn}(k) \sqrt{1-\frac{\left(C_{0}^{2}-U_{0}^{2}\right)}{\omega^{2}} \alpha_{|k|}^{2}(X)} .
$$

A cut-on cut-off transition point $[9,11]$ at $X=X_{t}$ for a given mode $n$ is defined to be where $\sigma_{n}^{2}\left(X_{t}\right)=\sigma_{-n}^{2}\left(X_{t}\right)=0$. At this axial location the $+n$ and $-n$ modal terms in Eq. (7) develop a singularity and so this solution is no longer valid. Further analysis of the left-hand side of (6) within an inner scaling region of the form $\left|X-X_{t}\right| \sim \epsilon^{2 / 3}$ leads to removal of the singularity and a solution satisfying Airy's equation. Hence, the slowly-varying mode $n$ interacts with its opposite-running counterpart resulting in total reflection of the mode. For such a mode undergoing cut-on cut-off transition inside a duct geometry, a uniformly valid composite solution [11] can be substituted in place of the modal component in Eq. (7), and this composite solution takes the form

$$
\hat{Q} \sqrt{\frac{C_{0}}{\omega D_{0}}} \psi_{|n|}(y, z ; X)\left(\frac{(-3)}{2 \epsilon \sigma_{n}^{3}} \int_{X_{t}}^{X} \frac{\omega C_{0} \sigma_{n}}{C_{0}^{2}-U_{0}^{2}} \mathrm{~d} X^{\prime}\right)^{\frac{1}{6}} \mathrm{Ai}\left[\left(\frac{3 \mathrm{i}}{2 \epsilon} \int_{X_{t}}^{X} \frac{\omega C_{0} \sigma_{n}}{C_{0}^{2}-U_{0}^{2}} \mathrm{~d} X^{\prime}\right)^{\frac{2}{3}}\right] \mathrm{e}^{\frac{\mathrm{i}}{\epsilon} \int_{X_{t}}^{X} \frac{\omega U_{0}}{\left(C_{0}^{2}-U_{0}^{2}\right)} \mathrm{d} X^{\prime}} .
$$

With the exception of this phenomenon, there are no other possible mechanisms for inter-modal scattering within the standard multiple-scales framework for $\omega \sim 1$ unless a sharp change in either geometry or boundary conditions is imposed; one example of this is scattering at an interface between an acoustically-hard duct wall and a lined finite-impedance duct wall $[14,15]$.

\section{High frequency analysis}

We assume now that the Helmholtz number is high, say $\omega \sim \epsilon^{-q}$ where $q>0$, and we substitute the slowly-varying mean flow expansion (1) into the governing wave equation (4) and examine the terms at all orders. The highest power of frequency in any term within the equation is $\omega^{2}$ and the highest derivative of $\phi$ present is second order. Hence, as seen in [4], we can obtain the following

$$
\begin{aligned}
(1 & \left.-\frac{U_{0}^{2}}{C_{0}^{2}}\right) \phi_{x x}+\nabla_{\perp}^{2} \phi+\frac{\omega^{2}}{C_{0}^{2}} \phi-\frac{2 \mathrm{i} \omega U_{0}}{C_{0}^{2}} \phi_{x} \\
= & -\epsilon\left\{D_{0}^{-1} \frac{\partial D_{0}}{\partial X} \phi_{x}-\mathrm{i} \omega U_{0} \frac{\partial}{\partial X}\left(\frac{1}{C_{0}^{2}}\right) \phi-U_{0} \frac{\partial}{\partial X}\left(\frac{U_{0}}{C_{0}^{2}}\right) \phi_{x}-\frac{2 \mathrm{i} \omega}{C_{0}^{2}}\left(\mathbf{V}_{\perp} \cdot \nabla_{\perp} \phi\right)\right. \\
& \left.-2 \frac{U_{0}}{C_{0}^{2}}\left(\mathbf{V}_{\perp} \cdot \nabla_{\perp} \phi_{x}\right)\right\}+O\left(\epsilon^{2} \omega^{2} U_{0}\right) .
\end{aligned}
$$

Avoiding cut-on cut-off transition points, a modal type outer solution similar to Eq. (7) can still be obtained for $\omega \gg 1$ from the convective wave equation within the duct, where $\alpha_{|k|}(X)$ and $\sigma_{k}$ are expansions in powers of $\epsilon$. However, it is around the singular transition points where $\sigma_{k}=0$ that previous analyses need some revision.

Around the axial location $X_{t}$ where a particular mode $n$ has $\sigma_{n}\left(X_{t}\right)=0$, an inner scaling region is necessary and the thickness of this region is determined by balancing the $\phi_{x x}$ term with the other terms on the left-hand side of Eq. (9). These other terms, by substituting $\nabla_{\perp}^{2} \phi=-\alpha_{|n|}^{2} \phi$, can be combined together and simplified as $\phi \omega^{2} C_{0}^{2} \sigma_{n}^{2}\left(C_{0}^{2}-U_{0}^{2}\right)^{-2}$, which tends to zero as we approach $X_{t}$. Approaching this limit, it balances $\phi_{x x}$ in the vicinity around the turning point when

$$
\frac{\epsilon^{2}}{\left|X-X_{t}\right|^{2}} \sim \omega^{2}\left|X-X_{t}\right| \text {. }
$$

Therefore the inner scaling region can be defined by $\left|X-X_{t}\right| \sim(\epsilon / \omega)^{2 / 3}$ and we note that this inner region shrinks in width as the Helmholtz number increases.

A much more important contrast from the $\omega \sim 1$ case, however, is that the last two non-parallel terms on the right-hand side of Eq. (9) become significantly larger as $\omega$ increases within the defined inner scaling region around a cut-on cut-off transition point. Hence, for $\omega \gg 1$, four specific terms can now potentially balance in the inner-region solution and they are (along with their relative scalings): 
term $1: \phi_{x x} \sim \frac{\epsilon^{2}}{\left|X-X_{t}\right|^{2}}$

term $2: \frac{\omega^{2} C_{0}^{2} \sigma_{n}^{2}}{\left(C_{0}^{2}-U_{0}^{2}\right)^{2}} \phi \sim \omega^{2}\left|X-X_{t}\right|$

$\operatorname{term} 3: \epsilon\left\{\frac{2 \mathrm{i} \omega}{C_{0}^{2}}\left(\mathbf{V}_{\perp} \cdot \nabla_{\perp} \phi\right)\right\} \sim \epsilon \omega \alpha_{|n|} U_{0}$,

term $4: \epsilon\left\{\frac{2 U_{0}}{C_{0}^{2}}\left(\mathbf{V}_{\perp} \cdot \nabla_{\perp} \phi_{x}\right)\right\} \sim \epsilon \omega \alpha_{|n|} U_{0}^{2}$,

where, in term $4, \phi_{x} \sim \omega U_{0}$ in line with the expected convective component which is cancelled out on the left-hand side. Terms 1 and 2 alone lead to Airy's equation for $\omega \sim 1$ when $X-X_{t} \sim \epsilon^{2 / 3}$ but we can conclude from our scalings that at least one of the non-parallel terms 3 and 4 driven by the crosswise mean velocity become comparable for frequencies of $O\left(\epsilon^{-1 / 2}\right)$ or higher-a scaling that is significantly lower than the previous estimate obtained [8]. Note that these additional terms require a mean flow to exist and their magnitude is determined by both the strength of the mean flow, assuming $U_{0}=O(1)$, and the magnitude of the cross-sectional eigenvalue $\alpha_{n}\left(X_{t}\right)$, which is assumed to be comparable to the Helmholtz number.

The inner scaling region around the cut-on cut-off transition point of the incident mode can be defined thus

$$
\left(X-X_{t}\right)=\left(\frac{\epsilon}{\omega}\right)^{2 / 3} \lambda_{0}^{-1} \xi
$$

where $\xi=O(1)$ is the inner scaled axial variable and $\lambda_{0}$ is a positive parameter given by

$$
\lambda_{0}^{3}=\frac{2 C_{0}^{2}}{\left(C_{0}^{2}-U_{0}^{2}\right)^{2}}\left(\frac{C_{0}\left(X_{t}\right) C_{0}^{\prime}\left(X_{t}\right)-U_{0}\left(X_{t}\right) U_{0}^{\prime}\left(X_{t}\right)}{C_{0}^{2}\left(X_{t}\right)-U_{0}^{2}\left(X_{t}\right)}+\frac{\alpha_{n}^{\prime}\left(X_{t}\right)}{\alpha_{n}\left(X_{t}\right)}\right) .
$$

Note that $\lambda_{0}$ is related to the parameter $\lambda$ given in Rienstra's previous work $[9,4]$ by a simple scaling, $\lambda=\omega^{2 / 3} \lambda_{0}$. We now assume that the inner region solution for the acoustic velocity potential takes the form

$$
\phi_{\text {inner }}=\sum_{k=0}^{\infty} \chi_{k}(\xi) \psi_{k}(y, z ; X) \mathrm{e}^{+\frac{i}{\epsilon} \int_{X_{t}}^{X} \frac{\omega U_{0}}{c_{0}^{2}-U_{0}^{2}} d X^{\prime}},
$$

and substituting this expression into the convective wave equation (9) we find to leading order that

$$
\epsilon^{2 / 3} \omega^{4 / 3} \lambda_{0}^{2} \sum_{k=0}^{\infty}\left[\frac{\mathrm{d}^{2} \chi_{k}}{\mathrm{~d} \xi^{2}}-\left(\xi+\delta_{k, n}\right) \chi_{k}\right] \psi_{k}\left(y, z ; X_{t}\right)=+\frac{2 \mathrm{i} \epsilon \omega C_{0}^{2}}{\left(C_{0}^{2}-U_{0}^{2}\right)^{2}} \sum_{k=0}^{\infty} \chi_{k} \mathbf{V}_{\perp} \cdot \nabla_{\perp} \psi_{k}\left(y, z ; X_{t}\right) .
$$

The parameter $\delta_{k, n}$ in the above equation represents the relative spacing of each neighbouring mode's $\sigma_{k}^{2}$ to the incident mode's $\sigma_{n}^{2}$ within the inner region. For the incident mode undergoing cut-on cut-off transition, $\delta_{n, n}=0$ so that the left-hand side of Eq. (12) reduces to the usual Airy's equation.

Eq. (12) contains a summation over all eigenmodes. However, the impact of the $\left\{\mathbf{V} \cdot \nabla_{\perp} \phi\right\}$ term on the right-hand side is that, even if the modes are orthogonal, it is no longer possible to decouple the individual modal equations as, on integrating over the duct cross-section $\mathscr{A}(X)$, we find typically that

$$
\iint_{\mathscr{A}(X)} \psi_{n}\left(\mathbf{V}_{\perp} \cdot \nabla_{\perp} \psi_{k}\right) \mathrm{d} y \mathrm{~d} z \neq 0 \quad \text { for } k \neq n .
$$

Therefore an incident mode undergoing cut-on cut-off transition can influence its neighbouring modes through a non-zero right-hand side. As a result, the new inner region solution consists of a series of coupled forced Airy's equation type problems along with the expected boundary conditions specifying any incoming waves, while suppressing spurious reflections of outgoing waves from the domain. It is important to observe that $\mathbf{V}_{\perp} \neq 0$ to induce this scattering mechanism and so it requires both a mean flow and a geometry variation leading to cut-on cut-off transition. The effect of the scattering mechanism can be most easily demonstrated in a duct of rectangular cross section and this is described in Section 7. However, it is important to note that the mechanism achieves a similar effect in cylindrical and more general cross-sectional geometries and such extensions are briefly detailed in the discussion at the end of the paper.

For situations with mean flow where $\omega \gg \epsilon^{-1 / 2}$, a balance between terms 1,2 and 3 remains (with term 4 negligible) so long as $U_{0} \sim \epsilon^{-1 / 3} \omega^{-2 / 3} \ll 1$. For $U_{0} \sim 1$ and higher frequencies the scattering terms ( 3 and 4 ) become much larger in magnitude and act over length scales significantly longer than the inner scaling region leading to stronger scattering. Note though that the left-hand side terms of Eq. (9) along with the two right-hand terms 3 and 4 identified remain the dominant terms in the governing equations at these higher frequencies until $\omega \sim \epsilon^{-2}$ where a second mechanism is introduced as described in the next section. 


\section{Modal scattering without mean flow}

Of course, scattering is still expected to occur at high frequencies even when a mean flow is not present, but clearly the mechanism defined in the previous section does not apply when $U_{0}=0$, and thus $\mathbf{V}_{\perp}=0$. For the no-flow case our governing equation (9) for the acoustic potential simply reduces to

$$
\phi_{x x}+\nabla_{\perp}^{2} \phi+\frac{\omega^{2}}{C_{0}^{2}} \phi=0
$$

exactly, where $C_{0}$ is now a constant. The only non-parallel effect remaining is the slowly varying geometry, the boundary condition of which determines the slowly-varying eigenvalues $\alpha_{k}(X)$.

Proceeding with an inner-region analysis we assume that (i) $\omega \gg 1$, (ii) the inner-region variable $\xi$ about the turning point is as defined in (10), and (iii) the acoustic potential takes the form

$$
\phi_{\text {inner }}=\sum_{k=0}^{\infty} \chi_{k}(\xi) \psi_{k}(y, z ; X),
$$

obviously now without the convection term. In this situation, any non-parallel effects can only arise from the partial differentiation of $\psi_{k}$ with respect to the slow variable $X$. It is expected that for $\omega \gg 1$ such partial differentiation leads to

$$
\begin{aligned}
& \frac{\partial \psi_{k}(y, z ; X)}{\partial X} \sim\left|\alpha_{k}^{\prime}(X) \psi_{k}\right|=O(\omega \epsilon), \\
& \frac{\partial^{2} \psi_{k}(y, z ; X)}{\partial X^{2}} \sim\left|\left(\alpha_{k}^{\prime}(X)\right)^{2} \psi_{k}\right|=O\left(\omega^{2} \epsilon^{2}\right),
\end{aligned}
$$

as $\alpha_{k} \sim \omega_{k}$ near a turning point; these terms have been negligible up until now in any previous analyses. As such, we expand $\phi_{x x}$ as follows,

$$
\phi_{x x}=\sum_{k=0}^{\infty} \frac{\epsilon^{2}}{\left|X-X_{t}\right|^{2}} \chi_{k}^{\prime \prime}(\xi) \psi_{k}+2 \frac{\epsilon}{\left|X-X_{t}\right|} \chi_{k}^{\prime}(\xi) \frac{\partial \psi_{k}}{\partial X}+\chi_{k}(\xi) \frac{\partial^{2} \psi_{k}}{\partial X^{2}},
$$

and determine that the three terms become comparable in the region $\left|X-X_{t}\right| \sim \omega^{-1}$. Balancing these terms with the remaining part of the governing equation near a turning point, given by

$$
\nabla_{\perp}^{2} \phi+\frac{\omega^{2}}{C_{0}^{2}} \phi \sim \omega^{2}\left|X-X_{t}\right|
$$

leads to $\left|X-X_{t}\right| \sim(\epsilon / \omega)^{2 / 3}$ as before and, as a result, the non-parallel terms above become comparable in the inner region when $\omega \sim \epsilon^{-2}$; this agrees exactly with the initial approximate estimate obtained previously [8]. Hence for the case $\omega=O\left(\epsilon^{-2}\right)$, substituting our expression for the inner solution (14) into (13) leads to

$$
\sum_{k=0}^{\infty}\left[\chi_{k}^{\prime \prime}(\xi)-\left(\xi+\delta_{k, n}\right) \chi_{k}(\xi)\right] \psi_{k}\left(y, z, X_{t}\right)=-\sum_{k=0}^{\infty}\left\{\left.\frac{2 \epsilon}{\lambda_{0}} \chi_{k}^{\prime}(\xi) \frac{\partial \psi_{k}}{\partial X}\right|_{X=X_{t}}+\left.\frac{\epsilon^{2}}{\lambda_{0}^{2}} \chi_{k}(\xi) \frac{\partial^{2} \psi_{k}}{\partial X^{2}}\right|_{X=X_{t}}\right\} .
$$

And, in a similar manner to the flow-induced scattering mechanism derived in the previous section, the fact that typically

$$
\iint_{\mathscr{A}(X)} \psi_{n} \frac{\partial \psi_{k}}{\partial X} \mathrm{~d} y \mathrm{~d} z \neq 0, \quad \iint_{\mathscr{A}(X)} \psi_{n} \frac{\partial^{2} \psi_{k}}{\partial X^{2}} \mathrm{~d} y \mathrm{~d} z \neq 0, \quad \text { for } k \neq n
$$

indicates that the equation derived here represents a second geometry-induced mechanism that becomes significant at much higher Helmholtz numbers.

\section{Scattering example in a rectangular duct}

It is simplest to demonstrate how the additional non-parallel terms induce intermodal scattering by taking the example of a slowly-varying duct of rectangular cross section defined by

$$
0<y<1, \quad 0<z<h(X), \text { for }-\infty<X<+\infty,
$$

with acoustically hard walls so that $\nabla \phi \cdot \mathbf{n}=0$, where $\mathbf{n}$ is the unit vector normal to the wall, at $y=0,1$ and $z=0, h(X)$. The remainder of this paper, including the numerical results of Section 9, analyses a duct geometry of this type. A uniform mean flow is present in the duct given by Eq. (2) and, in this case, the mean crossflow component reduces to

$$
\mathbf{v}_{\perp}(X, y, z)=\widehat{W}(X) z \mathbf{e}_{\mathbf{z}},
$$

with $\widehat{W}(X)$ known via the solution to Eqs. (2) and (3). A modal eigensolution to the cross-sectional eigenvalue problem is

$$
\psi_{k}(y, z ; X)=\frac{4}{h(X)} \cos \left[\beta_{k}(X) z\right] \cos (m \pi y),
$$


and, assuming the Helmholtz number to be large $\omega \gg 1$, we select a certain incident mode $p$ with $\beta_{p}(X)=p \pi / h(X)$ (satisfying the hard-wall boundary conditions to leading order) where $p$ and $m$ are integers large enough so that $\beta_{p}^{2}(X)+(m \pi)^{2}$ $\sim \omega^{2}$.

Computing the inner solution problem is not useful on its own without matching to the outer modal solution for $\left|X-X_{t}\right| \gg(\epsilon / \omega)^{2 / 3}$ far from the transition point of mode $p$ at $X_{t}$. Ideally, we would like to develop a composite solution as given in Eq. (8) for the flow-induced scattering problem. Following the analysis as done for the $\omega \sim 1$ case [11] leads to an equivalent stretched variable $s=\epsilon^{-\gamma} g(X)$ which is an integral of the reduced axial wavenumber for mode $p$. However, this creates some difficulties in expressing the $\sigma_{n}$ of the neighbouring modes $(n \neq t)$ in terms of the stretched variable $s$ leading to a rather laborious calculation. In short, the composite solution for the scattering problem no longer leads to a simple analytic form in terms of standard Airy functions and so some degree of numerical computation is unavoidable. With this in mind, we pursue a composite solution approach but with the aim to convert the problem to a coupled set of ordinary differential equations-one for every mode $n$ which can then be truncated and solved iteratively.

\section{Flow induced scattering when $\omega \sim \epsilon^{-1 / 2}$}

Based on the scaling obtained in Section 4 for the flow-induced mechanism, we initially rescale $\omega=\epsilon^{-1 / 2} \bar{\omega}$ and $\beta_{k}=\epsilon^{-1 / 2} \bar{\beta}_{k}$ where $\bar{\omega} \sim 1$ and $\bar{\beta}_{k} \sim 1$ with an arbitrary $m \lesssim \epsilon^{-1 / 2}$ constant wavenumber in the non-varying $y$-direction included for convenience. From the composite solution approach, we assume an acoustic velocity potential field of the form

$$
\phi=\sum_{k=0}^{\infty} \Phi(X) \chi_{k}(s) \cos \left(\epsilon^{-1 / 2} \bar{\beta}_{k}(X) z\right) \cos (m \pi y) \mathrm{e}^{\frac{\mathrm{i}}{\epsilon} \int_{X_{t}}^{X} \frac{\epsilon^{-1 / 2} \bar{\omega} U}{c^{2}-U^{2}} \mathrm{~d} X^{\prime}},
$$

where $s=\epsilon^{-q} X$, and substitute this into our governing equation (9). A careful scaling to preserve the inner scaling region equation obtained can be achieved by choosing $q=1$, and thus $s=x$, leading to

$$
\begin{aligned}
& \sum_{k=0}^{\infty} \cos \left(\epsilon^{-1 / 2} \bar{\beta}_{k}(X) z\right)\left[\chi_{k}^{\prime \prime}(x)+\epsilon^{-1} \frac{\omega^{2} C_{0}^{2} \sigma_{k}^{2}}{\left(C_{0}^{2}-U_{0}^{2}\right)^{2}} \chi_{k}(s)\right] \\
& +\frac{2 \mathrm{i} \bar{\omega} C_{0}^{2} \widehat{W}(X) z}{\left(C_{0}^{2}-U_{0}^{2}\right)^{2}} \sum_{k=0}^{\infty} \bar{\beta}_{k}(X) \chi_{k}(x) \sin \left(\epsilon^{-1 / 2} \bar{\beta}_{k}(X) z\right)=O\left(\epsilon^{1 / 2}\right),
\end{aligned}
$$

where, from the dispersion relation,

$$
\bar{\beta}_{k}^{2}(X)+\epsilon m^{2} \pi^{2}=\frac{\bar{\omega}^{2}}{\left(C_{0}^{2}-U_{0}^{2}\right)}\left(1-\sigma_{k}^{2}\right) .
$$

Multiplying Eq. (18) by $\cos \left(\epsilon^{-1 / 2} \bar{\beta}_{n}(X) z\right)$ and integrating in $z$ across the channel yields

$$
\chi_{n}^{\prime \prime}+\left(\frac{\epsilon^{-1} \bar{\omega}^{2} C_{0}^{2} \sigma_{n}^{2}}{\left(C_{0}^{2}-U_{0}^{2}\right)^{2}}\right) \chi_{n}=\frac{2 \mathrm{i} \bar{\omega} C_{0}^{2} \widehat{W}(X)}{\left(C_{0}^{2}-U_{0}^{2}\right)^{2}} \sum_{k=0}^{\infty} \mathcal{C}_{k, n} \bar{\beta}_{k}(X) h(X) \chi_{k},
$$

and a scattering coefficient $\mathcal{C}_{k, n}$ can now be identified as

$$
\mathcal{C}_{k, n}=\frac{(-2)}{h^{2}(X)} \int_{0}^{h(X)} z \sin \left(\beta_{k} z\right) \cos \left(\beta_{n} z\right) \mathrm{d} z=\left\{\begin{array}{cc}
\frac{1}{\pi}\left(\frac{(-1)^{k+n}}{(k+n)}+\frac{(-1)^{k-n}}{(k-n)}\right) & k \neq n \\
\frac{1}{2 \pi n} & k=n .
\end{array}\right.
$$

Note that, as in the $\omega \sim 1$ composite solution [11], a slowly varying coefficient $\Phi(X)$ is also needed and is determined by matching the solution from Eq. (18) to the outer solution given by Eq. (7). Away from any region of crosswise mean flow, $\chi_{n}(x)$ is the solution to

$$
\chi_{n}^{\prime \prime}+\left(\frac{\epsilon^{-1} \bar{\omega}^{2} C_{0}^{2} \sigma_{n}^{2}}{\left(C_{0}^{2}-U_{0}^{2}\right)^{2}}\right) \chi_{n}=0 .
$$

On matching this directly to the expected outer solution, we find

$$
\Phi(X)=\sqrt{\frac{C_{0}^{2}(X)}{\left[C_{0}^{2}(X)-U_{0}^{2}(X)\right] D_{0}(X) h(X)}},
$$

which is independent of the mode number. Hence we are now able to obtain from our numerical solution for $\chi_{n}$ the acoustic velocity potential for each mode $n$ from Eq. (17) and subsequently the acoustic pressure using the relation in (5). 


\section{Geometry-induced scattering with no mean flow}

For the no-flow case, we increase the nondimensional Helmholtz number and cross-sectional eigenvalue to $\omega=\epsilon^{-2} \bar{\omega}$ and $\beta_{k}=\epsilon^{-2} \bar{\beta}_{k}$ respectively, where the terms with bars are of order unity. Assuming an acoustic velocity potential in a similar form as the previous section,

$$
\phi=\sum_{k=0}^{\infty} \Phi(X) \chi_{k}(s) \cos \left(\epsilon^{-2} \bar{\beta}_{k}(X) z\right) \cos (m \pi y),
$$

we substitute this into Eq. (13) and preserve the inner region scaling by choosing $q=2$ so that $s=\epsilon^{-1} x$; thus

$$
\begin{aligned}
& \sum_{k=0}^{\infty} \cos \left(\epsilon^{-2} \bar{\beta}_{k}(X) z\right)\left[\chi_{k}^{\prime \prime}(s)+\epsilon^{-2} \frac{\bar{\omega}^{2}}{C_{0}^{2}} \sigma_{k}^{2} \chi_{k}(s)\right] \\
& =\sum_{k=0}^{\infty} 2 z \bar{\beta}_{k}^{\prime}(X) \chi_{k}^{\prime}(s) \sin \left(\epsilon^{-2} \bar{\beta}_{k}(X) z\right)+z^{2}\left(\bar{\beta}_{k}^{\prime}(X)\right)^{2} \chi_{k}(s) \cos \left(\epsilon^{-2} \bar{\beta}_{k}(X) z\right),
\end{aligned}
$$

with dispersion relation given by

$$
\bar{\beta}_{k}^{2}(X)+\epsilon^{4} m^{2} \pi^{2}=\frac{\bar{\omega}^{2}}{C_{0}^{2}}\left(1-\sigma_{k}^{2}\right) .
$$

As before, multiplying by $\cos \left(\epsilon^{-2} \bar{\beta}_{n}(X) z\right)$ and integrating across the channel in $z$ produces

$$
\chi_{n}^{\prime \prime}(s)+\epsilon^{-2} \frac{\bar{\omega}^{2}}{C_{0}^{2}} \sigma_{n}^{2} \chi_{n}(s)=\sum_{k=0}^{\infty} g_{k, n} h^{2}(X)\left(\bar{\beta}_{k}^{\prime}(X)\right)^{2} \chi_{k}(s)-2 \mathcal{C}_{k, n} h(X) \bar{\beta}_{k}^{\prime}(X) \chi_{k}^{\prime}(s),
$$

where $\mathcal{C}_{k, n}$ is already defined in (20) and the second scattering coefficient $g_{k, n}$ is given by

$$
\frac{2}{h^{3}(X)} \int_{0}^{h(X)} z^{2} \cos \left(\beta_{k}(X) z\right) \cos \left(\beta_{n}(X) z\right) \mathrm{d} z=\left\{\begin{array}{cl}
\frac{2}{\pi^{2}}\left(\frac{(-1)^{n+k}}{(n+k)^{2}}+\frac{(-1)^{k-n}}{(k-n)^{2}}\right) & k \neq n \\
\frac{1}{\pi^{2}}\left(\frac{1}{2 n^{2}}+\frac{\pi^{2}}{3}\right) & k=n .
\end{array}\right.
$$

The slowly-varying matching coefficient, $\Phi(X)$, remains the same as for the with-flow case in the previous section, although obviously with $U_{0}=0$, and both $D_{0}$ and $C_{0}$ constant.

\section{Numerical results}

Based on the asymptotic analysis developed above we now attempt some numerical calculations of modal scattering generated by the two mechanisms highlighted. First, Eqs. (19) and (23) are rescaled into the original nondimensional axial variable $x$ and the right-hand sides are combined leading to the following equation:

$$
\begin{aligned}
\chi_{n}^{\prime \prime}(x)+\left(\frac{\omega^{2} C_{0}^{2} \sigma_{n}^{2}}{\left(C_{0}^{2}-U_{0}^{2}\right)^{2}}\right) \chi_{n}(x)= & h(x)\left\{\sum_{k=0}^{\infty} \frac{2 \mathrm{i} \omega C_{0}^{2} \widehat{W}}{\left(C_{0}^{2}-U_{0}^{2}\right)^{2}} \mathfrak{C}_{k, n} \beta_{k}(x) \chi_{k}(x)\right. \\
& \left.+\sum_{k=0}^{\infty} g_{k, n} h(x)\left(\beta_{k}^{\prime}(x)\right)^{2} \chi_{k}(x)-2 \mathcal{C}_{k, n} \beta_{k}^{\prime}(x) \chi_{k}^{\prime}(x)\right\} .
\end{aligned}
$$

Eq. (24) can also be obtained from (9) using the form suggested in (17) whilst keeping the right-hand side terms identified as dominant for scattering in the asymptotic analyses in Sections 7 and 8.

Our scattering model, Eq. (24), is computed for a finite set of modes $n=1, \ldots, N$ as a system of finite-differenced ordinary differential equations in parallel using a standard Thomas algorithm. A narrowing channel of the form

$$
h(x)=1-\frac{\Delta}{2}[1+\tanh (\kappa x)],
$$

is used as the duct geometry in the examples presented, where $\Delta$ and $\kappa$ are given constants and the multiple-scales small parameter $\epsilon$ can be expressed as $\epsilon=|\kappa \Delta / 2|$. The mean flow is set by choosing a value for the flux parameter $\mathcal{F}$ with the other mean flow parameter $\mathcal{E}=1 /(\gamma-1)$ fixed for all cases shown here. The parameter $\mathcal{F}$ can therefore be understood to represent the signed mean flow at $x=-\infty$, i.e. $\mathcal{F}=U_{0}(-\infty)$. For each computed mode $n$, non-reflecting boundary conditions are chosen at the extremities of the numerical domain $x_{\min }<x<x_{\max }$ of the form

$$
\begin{aligned}
& \chi_{n}^{\prime}-\mathrm{i} k_{n}^{-} \chi_{n}=-2 \mathrm{i} k_{n}^{-} \mathcal{A}_{n} \quad \text { at } x=x_{\min }, \\
& \chi_{n}^{\prime}+\mathrm{i} k_{n}^{+} \chi_{n}=2 \mathrm{i} k_{n}^{+} \mathcal{B}_{n} \quad \text { at } x=x_{\max },
\end{aligned}
$$



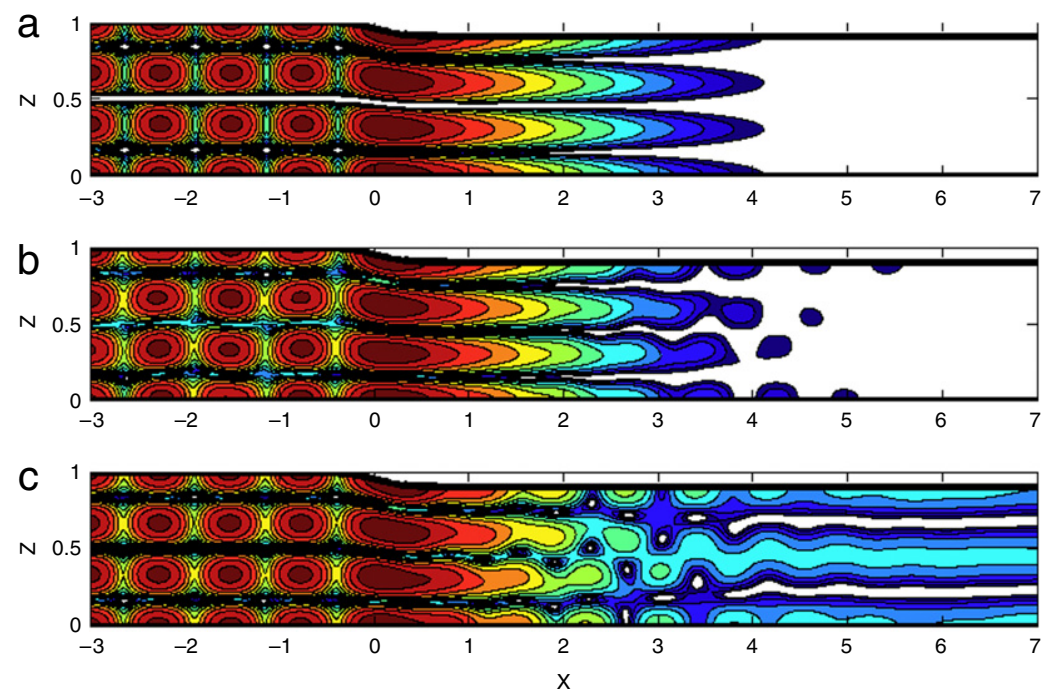

Fig. 1(a)-(c). Case A: $\omega=9.6, n=3$ incident from $x=-\infty$, and $\mathcal{F}= \pm 0.3$. Contour plot of the overall relative sound pressure level in the duct, given by $20 \log _{10}(|p(x, z)| / \max |p(x, z)|)$. Each contour level represents a change of $3 \mathrm{~dB}$. (a) is obtained from standard multiple-scales theory for $\mathcal{F}= \pm 0.3$; (b) is obtained from the scattering model in Eq. (24) for $\mathcal{F}=+0.3$; (c) is obtained from the scattering model in Eq. (24) for $\mathcal{F}=-0.3$.

with $k_{n}^{-}$and $k_{n}^{+}$equal to $\omega C_{0} \sigma_{n} /\left(C_{0}^{2}-U_{0}^{2}\right)$ at $x=x_{\min }$ and $x=x_{\max }$ respectively, and also where $\mathcal{A}_{n}$ and $\mathcal{B}_{n}$ represent the complex amplitudes of a mode incident from either $x=-\infty$ or $x=+\infty$ respectively. For the cases presented here all incident modes are right-running from $x=-\infty$ and thus $\mathcal{B}_{n} \equiv 0$ for all $n$. The boundaries $\left(x_{\min }, x_{\max }\right)$ of the computational domain are selected far enough away from the narrowing region where $W(X, z) \neq 0$ so as to suppress any unphysical phenomena from the finite-domain contaminating the solution obtained.

The finite number of modes $N$ computed for each case includes all the modes that are cut-on $\left(\sigma_{n}\right.$ real $)$ somewhere in the channel as well as the first few modes that are fully cut-off throughout the duct. An incident mode is chosen propagating from $x=-\infty$, so that $\mathcal{A}_{p} \neq 0$ for some $p$, and the solution is obtained iteratively by initially calculating the uncoupled problem with the right-hand side of (24) identically zero to obtain a first guess without scattering, $\chi_{n}^{(1)}$. This initial $\chi_{n}^{(1)}$ is then used to calculate a non-zero right-hand side of Eq. (24) before re-computing (24) to obtain a further guess, $\chi_{n}^{(2)}$. The process is then repeated a number of times until the absolute error between subsequent guesses $\chi_{n}^{(M)}$ and $\chi_{n}^{(M-1)}$ for every mode is below a certain prescribed tolerance. Our computations thus far indicate that only a handful of iterations, $M \sim 10$, is required to obtain extremely good convergence. Aside from the plots of the overall sound field and modal amplitudes presented, tables are also included that show the magnitudes of the reflected coefficient $\mathcal{R}_{n}$ and transmitted coefficient $\mathcal{T}_{n}$ for each mode $n$. These are the modal amplitudes for each mode $n$ scaled by the incident modal amplitude and travelling out of the numerical domain towards $x=-\infty$ and $x=+\infty$ respectively.

\subsection{Case $A: \epsilon=0.15, \omega=9.6,(n, m)=(3,0)$ incident}

The first case attempts to validate the dominance of the flow-induced scattering mechanism presented in Section 7 at moderately high frequencies. The geometry parameters chosen are $\kappa=3$ and $\Delta=0.1$ leading to a small parameter value of $\epsilon=0.15$. A non-dimensional frequency of $\omega=9.6$, lying in the range $\epsilon^{-1 / 2} \ll \omega \ll \epsilon^{-2}$, is selected, for which the theory predicts observable scattering only in cases where a mean flow is present in the duct. This first example is for a two-dimensional incident mode (the wavenumber in the $y$-direction $m=0$ ) and we find the first two modes $n=1,2$ are cut-on throughout the duct, whereas mode $n=3$ is cut-on (and thus propagates) only in the wider half of the duct for $x \ll 0$. Thus we set mode $n=3$ incident from $x=-\infty$ so that $\mathcal{A}_{3}=1$ and $\mathcal{A}_{n \neq 3}=0$. Eq. (24) is then computed for three scenarios: (i) for a mean flow moving left-to-right where $\mathcal{F}=+0.3$; (ii) a mean flow moving in the opposite direction with the same magnitude so $\mathcal{F}=-0.3$ and (iii) no mean flow, $\mathcal{F}=0$. The results obtained for these scenarios are presented in Figs. 1(a)-(f).

From standard $\omega \sim 1$ multiple-scales theory [9,11], the incident $n=3$ mode should simply reflect within the duct narrowing at the axial plane $X=X_{t}$ where $\sigma_{3}\left(X_{t}\right)=0$, resulting in a reflected mode of equal amplitude. A standing wave is thus expected in the region $X<X_{t}$ and no acoustic energy propagates beyond the transition point $X>X_{t}$. This prediction with no scattering is depicted in Fig. 1(a) in the form of a contour plot of the relative sound pressure level, given by $20 \log _{10}(|p(x, z)| / \max |p(x, z)|)$. The amplitude of the standing wave is identical for both cases $\mathcal{F}= \pm 0.3$ as the mean flow direction only affects the phase as seen in the uniformly valid composite solution of [11] given in Eq. (8). In contrast, relative sound pressure levels obtained from our scattering model (24) are shown 

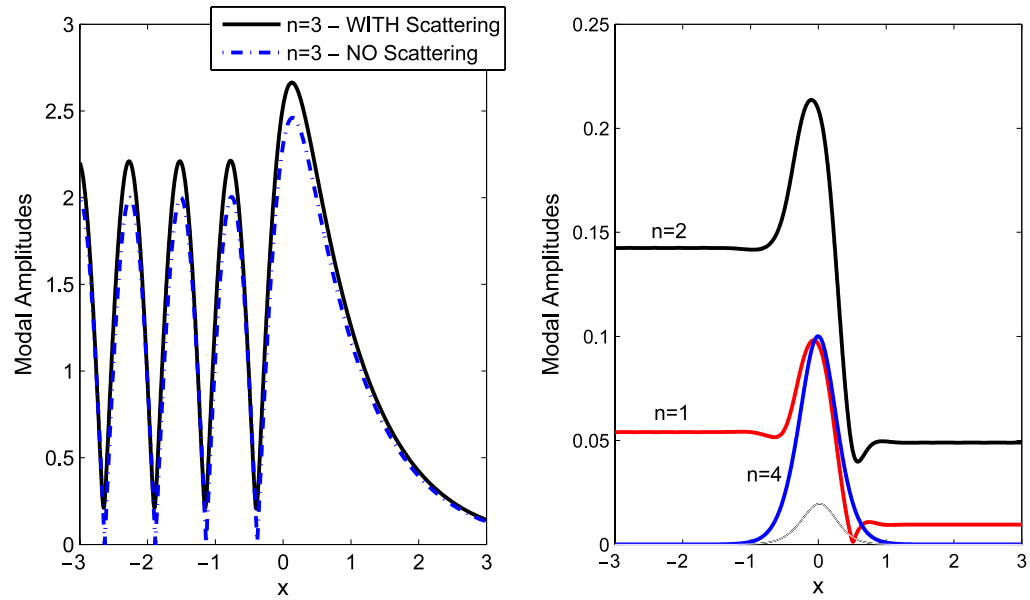

Fig. 1(d). Case A: $\omega=9.6, n=3$ incident, and $\mathcal{F}=+0.3$ (mean flow from left to right). The left plot shows the amplitude for the incident mode obtained from the scattering model (solid line) compared to the expected standing wave from the $\omega \sim 1$ multiple scales theory (dotted line). The right plot shows the scattered amplitudes for the neighbouring modes.
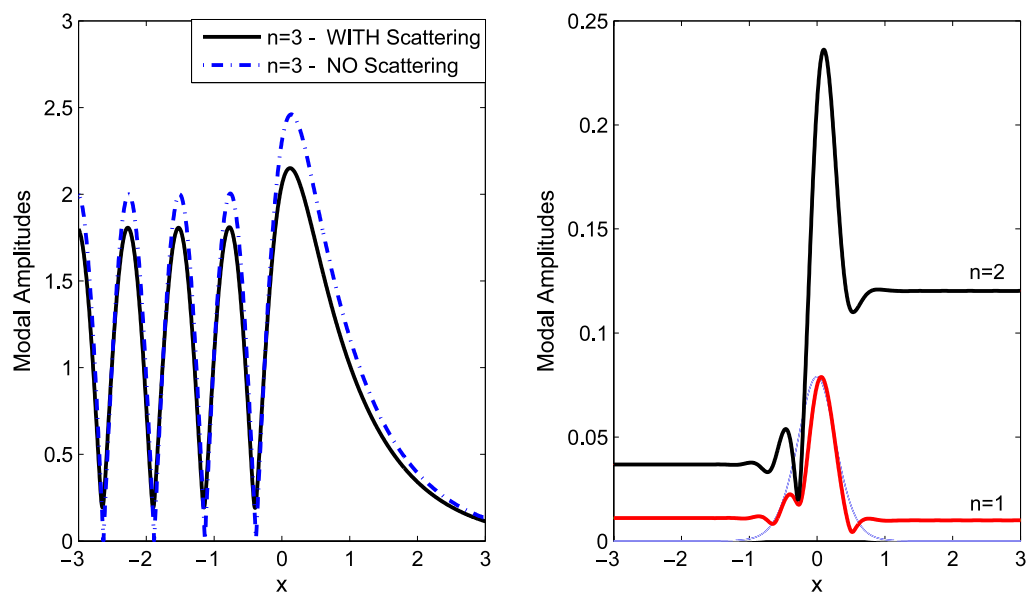

Fig. 1(e). Case A: $\omega=9.6, n=3$ incident, and $\mathcal{F}=-0.3$ (mean flow from right to left). The left plot shows the amplitude for the incident mode obtained from the scattering model (solid line) compared to the expected standing wave from the $\omega \sim 1$ multiple scales theory (dotted line). The right plot shows the scattered amplitudes for the neighbouring modes.

in Figs. 1(b) and 1(c) for scenarios $\mathcal{F}=+0.3$ and $\mathcal{F}=-0.3$ respectively. Figs. $1(\mathrm{~d})$ and $1(\mathrm{e})$ subsequently show the actual modal amplitudes, $\left|\Phi(x) \chi_{n}(x)\right|$, along the duct obtained from the scattering model. The left-hand plot in each of Figs. 1(d) and 1(e) shows the amplitude for the $n=3$ mode compared directly to the $\omega \sim 1$ standing wave theory and the right-hand plot in each figure shows the neighbouring scattered modal amplitudes. The main striking effect of the scattering model is that the neighbouring cut-on modes appear to have been excited in the narrowing region of the duct, where $h^{\prime}(x) \neq 0$, resulting in the generation of a significant $n=2$ scattered mode that propagates most of its scattered energy towards $x=-\infty$ when $\mathcal{F}>0$ and towards $x \rightarrow+\infty$ when $\mathcal{F}<0$. As a consequence, particularly for the $\mathcal{F}<0$ scenario, some acoustic energy is able to propagate beyond the transition point $X>X_{t}$ when the two scattering mechanisms identified are incorporated into the theory.

A second important observation from the scattering model is a marked change in the reflected component of the incident mode. For the $\mathcal{F}>0$ scenario, the left-hand plot of Fig. 1(d) suggests that the reflected mode computed is larger in magnitude than the incident amplitude (i.e. over-reflection) whereas for the reversed mean flow scenario $(\mathcal{F}<0)$ we see from Fig. 1(e) that the reflected $n=3$ component is actually diminished, becoming lower in amplitude than the incident. In each of the cases the absolute value of the reflection coefficient of incident mode, $\mathcal{R}_{3}$, (defined earlier as the ratio of reflected amplitude to incident amplitude) is calculated to be $\left|\mathcal{R}_{3}\right|=1.21$ and $\left|\mathcal{R}_{3}\right|=0.81$ for $\mathcal{F}=+0.3$ and $\mathcal{F}=-0.3$ respectively. This noteworthy alteration in the standing wave throws up some immediate questions regarding conservation of acoustic energy, something that has been up until now usually taken for granted in multiple-scales analyses for nearly uniform plug flow. For instance, clearly for the situation $\mathcal{F}>0$ where $\left|\mathcal{R}_{3}\right|>1,\left|\mathcal{R}_{n \neq 3}\right|>0$ and $\left|\mathcal{T}_{n \neq 3}\right|>0$, an increase in the acoustic energy must have occurred and this could only be generated by an interaction with the mean flow inside the duct. 

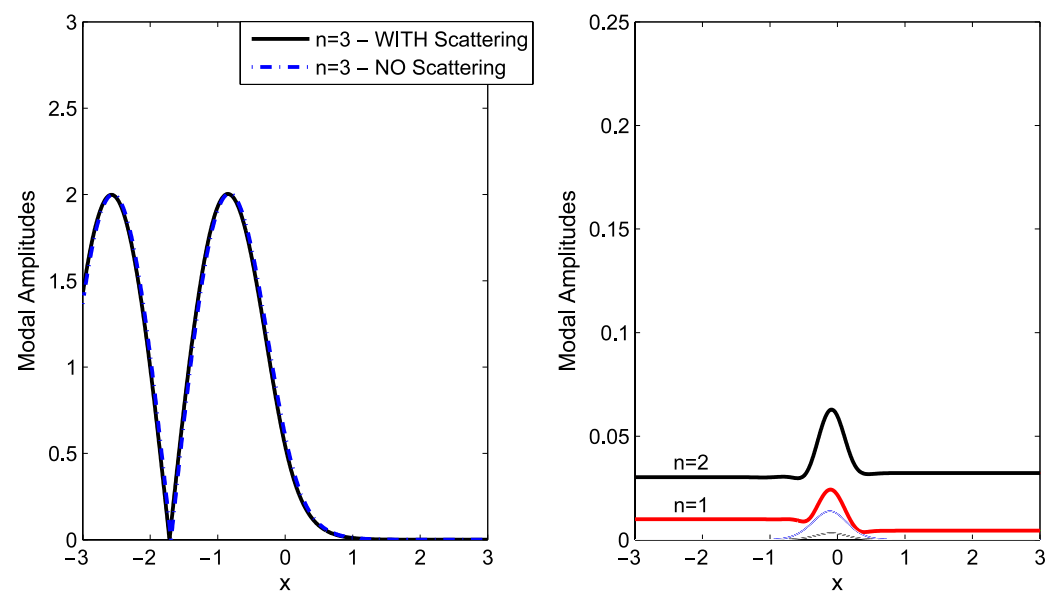

Fig. 1(f). Case A: $\omega=9.6, n=3$ incident, with no mean flow $\mathcal{F}=0$. The left plot shows the amplitude for the incident mode obtained from the scattering model (solid line) compared to the expected standing wave from the $\omega \sim 1$ multiple scales theory (dotted line). The right plot shows the scattered amplitudes for the neighbouring modes.

To quantify the overall change in acoustic energy due to interaction with the mean flow we calculate the acoustic power at either end of the duct domain and take the difference. The acoustic power through the duct cross-section at an axial location $x$ can be found by integrating the time-averaged acoustic intensity I [16] over the cross-section as follows

$$
\begin{aligned}
\mathcal{P}(x) & =\int_{0}^{1} \int_{0}^{h(x)} \mathbf{I} \cdot \mathbf{e}_{\mathbf{x}} \mathrm{d} z \mathrm{~d} y \\
& =\frac{1}{2} \int_{0}^{1} \int_{0}^{h(x)} \operatorname{Re}\left[\left(\frac{p}{D}+U_{0} \frac{\partial \phi}{\partial x}\right)\left(D \frac{\partial \phi}{\partial x}+\frac{p}{C^{2}} U_{0}\right)^{*}\right] \mathrm{d} z \mathrm{~d} y .
\end{aligned}
$$

A flow-induced power coefficient $\mathscr{E} \mathcal{P}$, which measures the additional power extracted from the mean flow, can be defined as follows

$$
\mathcal{E P}=\frac{\mathcal{P}\left(x_{\max }\right)-\mathcal{P}\left(x_{\min }\right)}{\mathcal{P}_{\text {incident }}},
$$

and here the coefficient is scaled here by $\mathcal{P}_{\text {incident }}$ which is the axial power of the incident mode alone at $x=-\infty$. For the $\mathcal{F}=-0.3$ case, where the reflection coefficient of the $n=3$ mode is less than unity, we find that $\mathscr{P}=-0.32$, indicating that the overall acoustic power has been reduced by almost a third. However, from the solution for $\mathcal{F}=+0.3$ we obtain $\mathscr{E P}=+0.52$, and so for the mean flow direction reversed the overall acoustic power has increased by over fifty percent.

So far we have concentrated on scenarios with a mean flow because, at this frequency, only significant flow-induced scattering is predicted by our theory. For comparison Fig. 1(f) shows the modal amplitudes obtained for the same setup as above but without mean flow $(\mathcal{F}=0)$. As predicted by the asymptotic analysis, one can observe that scattering is almost negligible without mean flow and, for the $n=3$ incident mode, the usual standing wave is practically unaltered.

\subsection{Case $B: \epsilon=0.2, \omega=40.6, m=0$}

Case B remains two-dimensional, $m=0$, but has a much higher Helmholtz number and a slightly steeper geometry variation with $\Delta=0.1$ and $\kappa=4$ so that $\omega \sim \epsilon^{-2}$; according to the asymptotic theory this should lead to significant scattering even when no mean flow is present due to the slow geometry variation. For $\mathcal{F}=0$, eleven modes are cut-on throughout the duct with mode $n=12$ undergoing cut-on cut-off transition at the duct narrowing. Therefore mode $n=12$ is chosen as the incident mode emanating from $x=-\infty$, thus $\mathscr{A}_{12}=1$ and $\mathcal{A}_{n \neq 12}=0$, and the relative sound pressure levels both for standard multiple-scales theory and Eq. (24) are shown in Figs. 2(a) and 2(b) respectively. Fig. 2(c) then shows the scattering amplitudes obtained which one can observe are now significantly larger than in case A. Without any mean flow, some of the acoustic field is scattered into the neighbouring mode $n=11$ generating a modal amplitude of magnitude close to 0.3 , with a smaller degree of scattering into modes $n=10$ and $n=9$. For the incident mode the reflected wave is slightly diminished, $\left|\mathcal{R}_{12}\right|=0.92$. This makes perfect sense as acoustic energy must be conserved if no mean flow is present and some energy has clearly been scattered into the neighbouring cut-on modes. Conservation of acoustic energy can be confirmed by calculating $|\mathcal{E} \mathcal{P}|$ which has a magnitude of less than $1 \%$ of the incident power; this can be regarded as well within the range of numerical error. The relative power transmitted through the right-end of the duct $\mathcal{P}\left(x_{\max }\right)$ is $11 \mathrm{~dB}$ lower than the incident power. 

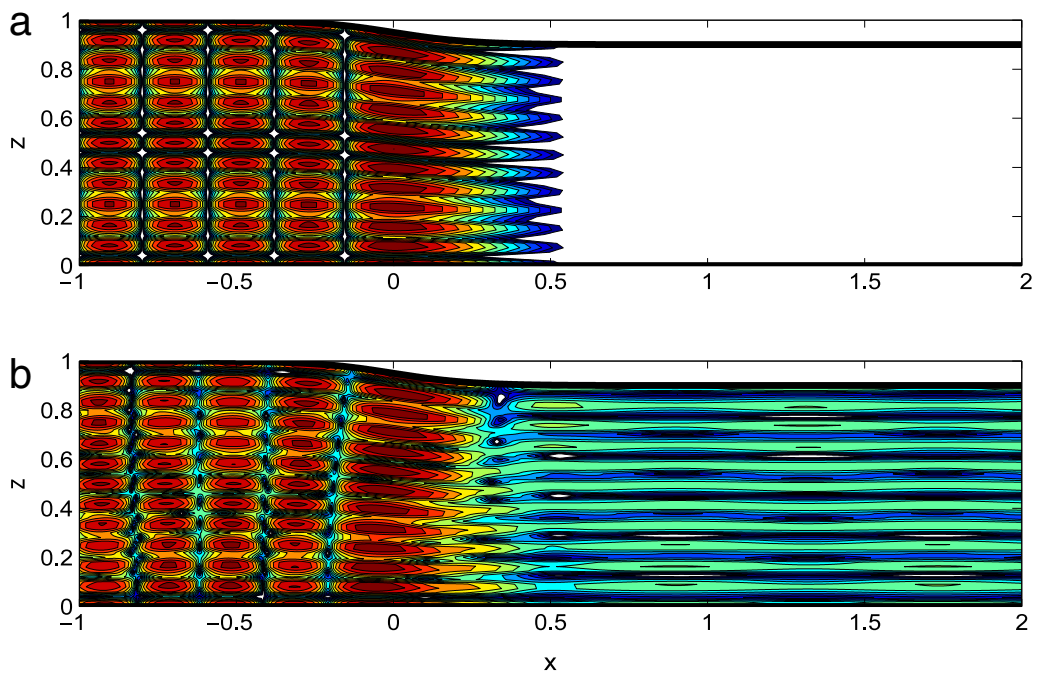

Fig. 2(a)-(b). Case B: $\omega=40.6,(n, m)=(12,0)$ incident from $x=-\infty$, with no mean flow. Contour plot of the overall relative sound pressure level in the duct, given by $20 \log _{10}(|p(x, z)| / \max |p(x, z)|)$. Each contour level represents a change of $3 \mathrm{~dB}$. (a) is obtained from standard multiple-scales theory for no flow and (b) is obtained from the scattering model in Eq. (24) for no flow.
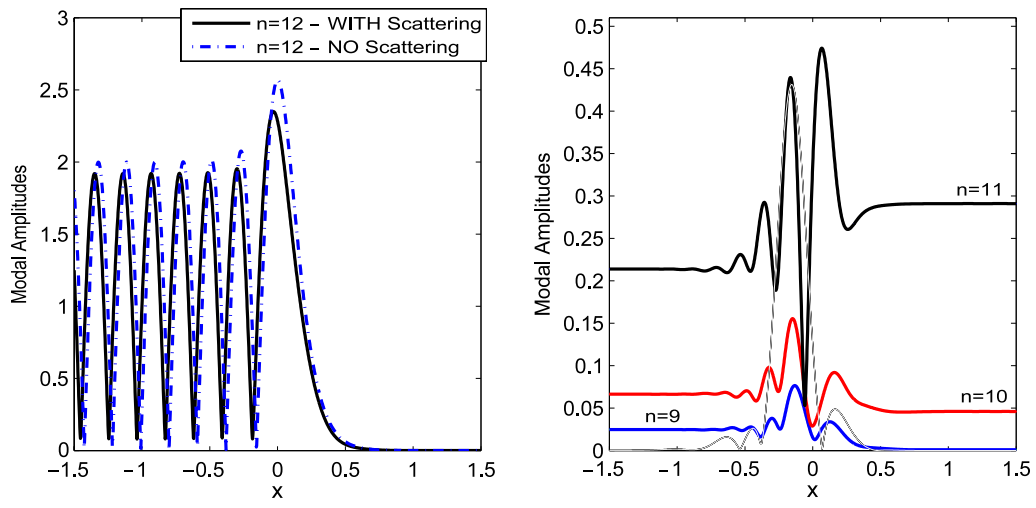

Fig. 2(c). Case B: $\omega=40.6,(n, m)=(12,0)$ incident, with no mean flow $\mathcal{F}=0$. The left plot shows the amplitude for the incident mode obtained from the scattering model (solid line) compared to the expected standing wave from the $\omega \sim 1$ multiple scales theory (dotted line). The right plot shows the scattered amplitudes for the neighbouring modes.

For comparison, we show here the same case but now with a mean flow and specifically for $\mathcal{F}= \pm 0.4$. For this level of mean flow, modes $n=1-13$ are cut-on throughout the duct with $n=14$ undergoing cut-on cut-off transition at the narrowing. Hence, mode $n=14$ is now selected as the incident mode from $x=-\infty$ instead. Modal amplitudes for $\mathcal{F}=-0.4$ and $\mathcal{F}=+0.4$ obtained from the solution to Eq. (24) are presented in Figs. 2(d) and 2(e), with a summary of reflected and transmitted coefficients presented in Table 1 . First note that the transmitted amplitudes $\widetilde{\mathcal{T}}_{n<14}$ are this time comparable to the no-flow case above, although there is significantly more of a scattered energy cascade beyond the nearest neighbour modes when $\mathcal{F}<0$, along with a lower reflection coefficient for the incident mode $\left|\mathcal{R}_{14}\right|=0.53$. However, for $\mathcal{F}>0$ much larger reflected modes are predicted and now over-reflection occurs, $\left|\mathcal{R}_{14}\right|=1.55$, and there is an $n=13$ left-running mode of amplitude $\left|\mathcal{R}_{13}\right|=0.48$. As might be expected, the interaction with the mean flow and exchange of energy is more significant than seen in case A due to the higher nondimensional frequency and we find that $\mathscr{E P}=-0.58$ when $\mathcal{F}=-0.4$ and $\mathcal{E} \mathcal{P}=1.89$ when $\mathcal{F}=+0.4$. Thus when $\mathcal{F}=+0.4$ the acoustic power is almost trebled although only $7 \%$ of the incident power $(-11.5 \mathrm{~dB})$ is transmitted beyond $x=x_{\max }$. For the situation where $\mathcal{F}=-0.4$, we find that $11 \%(-9.6 \mathrm{~dB})$ of the incident power is transmitted beyond the duct narrowing towards $x=+\infty$.

Given the observations from cases A and B presented so far it is possible to postulate that a contracting mean flow, where the mean flow accelerates through a narrowing, appears to inject energy into the acoustic field. A diverging flow on the other hand that slows as the duct widens extracts energy from the acoustic field. However, it would also appear in the current examples that although the acoustic power is increased by an accelerating mean flow through a duct narrowing, this additional power appears to be mainly reflected back through the wider section. For a two-dimensional incident mode or no flow case, propagating modes only become cut-off through a constriction and so this additional power seems unlikely to be trans- 

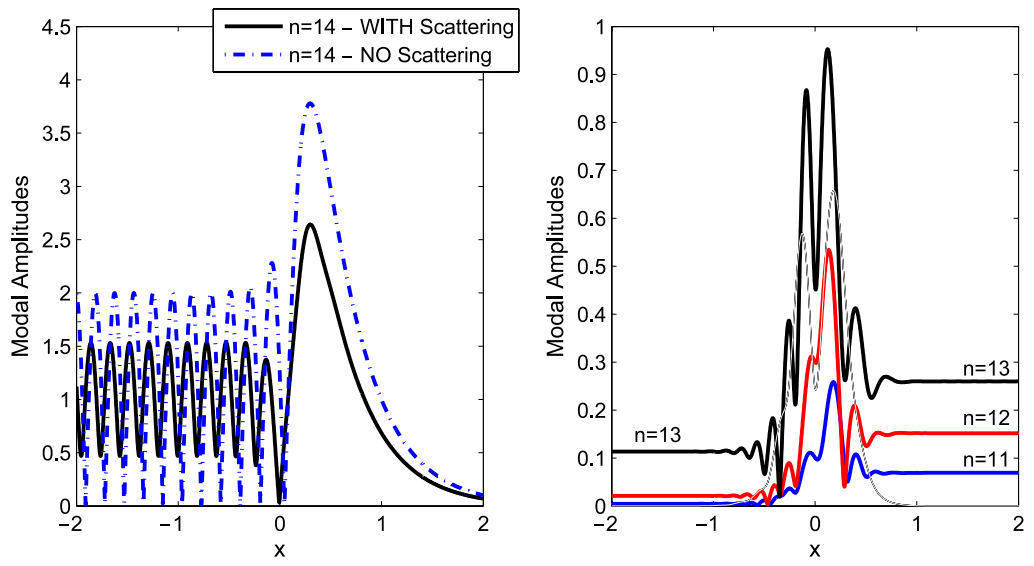

Fig. 2(d). Case B: $\omega=40.6,(n, m)=(14,0)$ incident, with $\mathcal{F}=-0.4$. The left plot shows the amplitude for the incident mode obtained from the scattering model (solid line) compared to the expected standing wave from the $\omega \sim 1$ multiple scales theory (dotted line). The right plot shows the scattered amplitudes for the neighbouring modes.
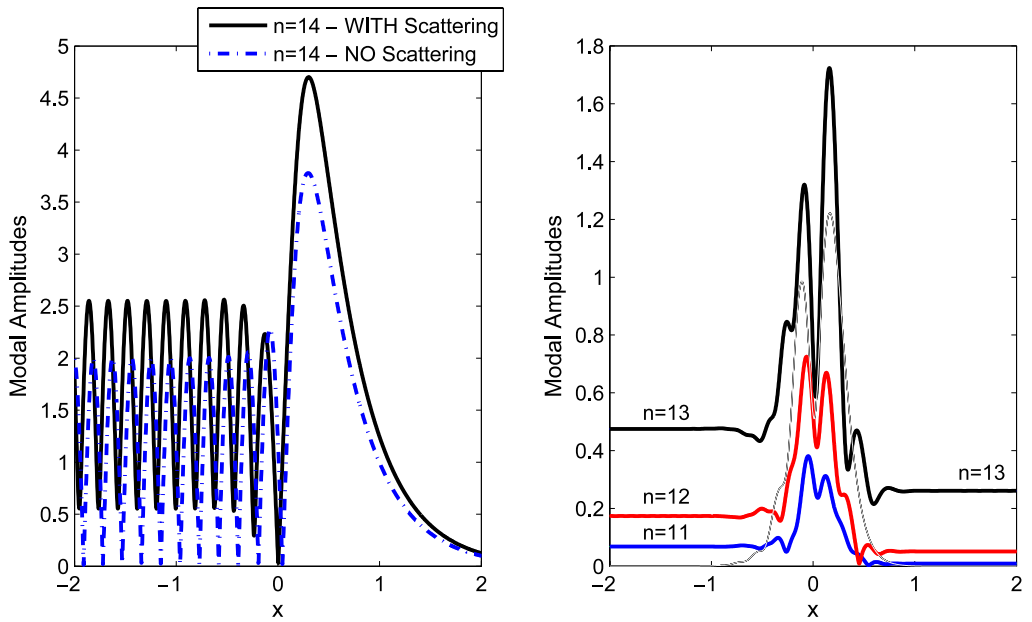

Fig. 2(e). Case B: $\omega=40.6,(n, m)=(14,0)$ incident, with $\mathcal{F}=+0.4$. The left plot shows the amplitude for the incident mode obtained from the scattering model (solid line) compared to the expected standing wave from the $\omega \sim 1$ multiple scales theory (dotted line). The right plot shows the scattered amplitudes for the neighbouring modes.

mitted beyond the transition point in such situations. However, the case below, where the $y$-wavenumber $m \neq 0$, shows a situation where this additional energy obtained from the mean flow can indeed be transmitted with rather dramatic results.

\subsection{Case $C: \epsilon=0.15, \omega=40,(n, m)=(4,14)$ incident}

This final example is for a similar Helmholtz number as in case B but with the more gradually varying duct of case A so that $\epsilon=0.15$. However, for this case we introduce some three-dimensionality by selecting a non-trivial eigenmode in the non-varying $y$-direction so that $m \neq 0$. For a large enough $m$, such as $m=14$ chosen here, and for a large enough mean flow the sign of $\mathrm{d} \sigma_{n}^{2} / \mathrm{d} x$ changes for the first few $n$ modes to be opposite to the sign of $h^{\prime}(x)$ unlike that seen in the $m=0$ cases. As a consequence, mode $(n, m)=(4,14)$, while cut-off for $h(x)=1$, becomes cut-on in a narrower duct where $h=0.9$. Fig. 3(a) shows the duct in case A, but reversed in $x(\Delta=0.1, \kappa=-3)$, and the standing wave generated by mode $(n, m)=(4,14)$ incident from $x=-\infty$ for $|\mathcal{F}|=0.4$ as predicted by standard $\omega \sim 1$ multiple-scales theory. Below this contour plot, Figs. 3(b) and 3(c) show the relative sound pressure levels obtained by our scattering model (24) for $\mathcal{F}=+0.4$ and $\mathcal{F}=-0.4$ respectively. Figs. 3(d) and 3(e) then show the modal amplitudes for the first few $(n, 14)$ modes in each of these mean flow cases and Table 2 summarises the reflected and transmitted modal amplitudes obtained.

This case confirms some of the conclusions drawn from cases A and B, and principally that a constricting flow accelerating through a narrowing duct injects energy into the scattered acoustic field, whereas a diverging slowing mean flow reduces the overall acoustic energy. However, in this case the reversal of the duct geometry leads to a very significant outcome in the $\mathcal{F}=-0.4$ case that the transmitted power $\mathcal{P}\left(x_{\max }\right)>\mathcal{P}_{\text {incident }}$. This situation is at complete odds to the standard multiple-scales theoretical prediction as, not only has acoustic energy leaked beyond the cut-on cut-off transition point, but 

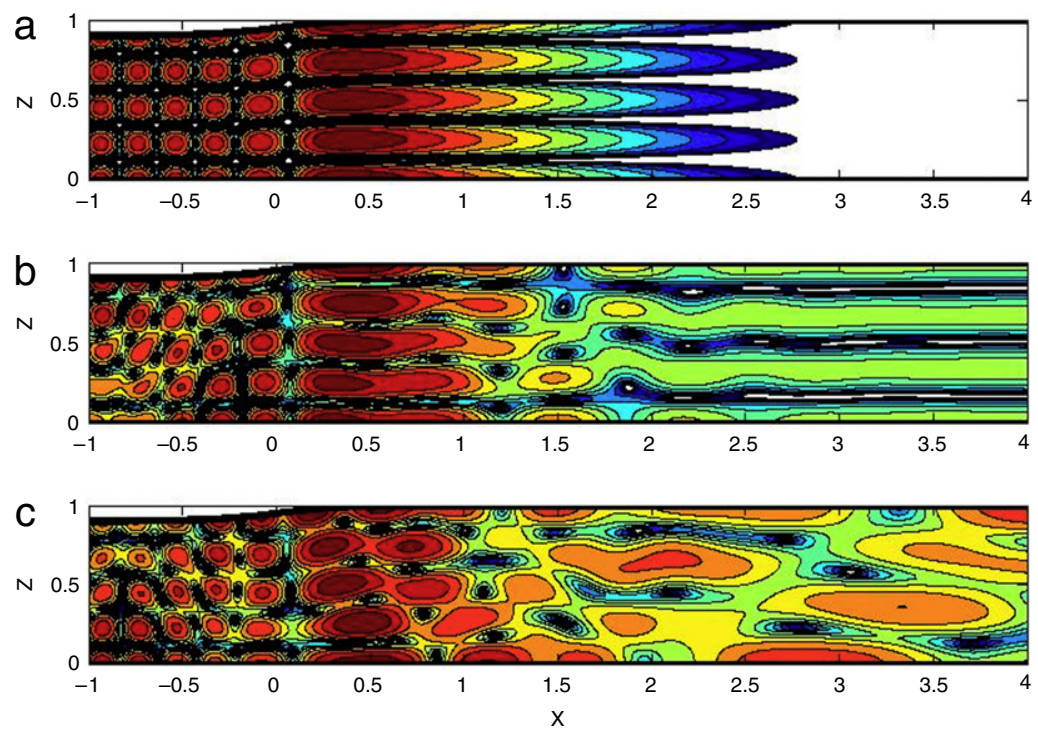

Fig. 3(a)-(c). Case C: $\omega=40,(n, m)=(4,14)$ incident from $x=-\infty$, with $\mathcal{F}= \pm 0.4$. Contour plot of the overall relative sound pressure level in the duct, given by $20 \log _{10}(|p(x, z)| / \max |p(x, z)|)$. Each contour level represents a change of $3 \mathrm{~dB}$. (a) is obtained from standard multiple-scales theory; (b) is obtained for $\mathcal{F}=+0.4$ from the scattering model in Eq. (24); (c) is obtained for $\mathcal{F}=-0.4$ from the scattering model in Eq. (24).
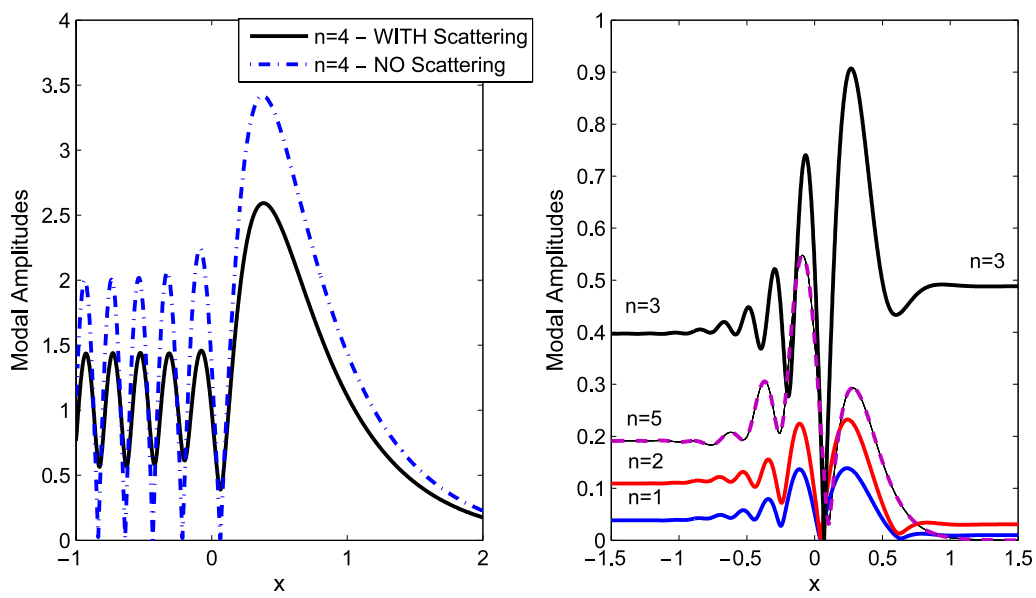

Fig. 3(d). Case C: $\omega=40,(n, m)=(4,14)$ incident, with $\mathcal{F}=+0.4$. The left plot shows the amplitude for the incident mode obtained from the scattering model (solid line) compared to the expected standing wave from the $\omega \sim 1$ multiple scales theory (dotted line). The right plot shows the scattered amplitudes for the neighbouring modes.

the acoustic energy transmitted is larger than that carried by the incident wave from $x=-\infty$. Indeed, $\mathcal{P}\left(x_{\max }\right)=1.36$ or, in other words, the acoustic power transmitted is $+1.36 \mathrm{~dB}$ higher than the incident power, although this amplified acoustic energy is now distributed between modes $n=1-3$. With regard to the defined flow-induced power coefficient, for $\mathcal{F}=-0.4, \mathcal{E P}=2.18$ meaning the acoustic power has more than trebled overall with the majority scattered forward and transmitted towards $x=+\infty$. In the case of the widening decelerating mean flow case $\mathcal{F}=+0.4$ we have $\mathscr{E P}=-0.41$ although the transmitted power is actually only $-7.70 \mathrm{~dB}$ lower than the incident power. It is interesting in addition to observe that mode $(n, m)=(5,14)$, which also undergoes cut-on cut-off transition in the duct, is excited in both flow regimes and is scattered backwards with a modal amplitude larger than that of modes $(1,14)$ and $(2,14)$.

Before concluding, it is worth just comparing the above with the predicted scattering obtained from a similar case without mean flow. For such a case, when $\mathcal{F}=0$, the modal amplitudes obtained are shown in Fig. 3(f). Of course, without a mean flow cut-on cut-off transition can only occur in a narrowing duct so we must revert back to exactly the duct used in case A, and hence with $\kappa=+3$. The Helmholtz number $\omega$ must also be raised slightly to keep mode $(n, m)=(4,14)$ cut-on for $x \ll 0$ and cut-off for $x \gg 0$. Notice that the scattered amplitudes obtained by the geometry-variations alone 

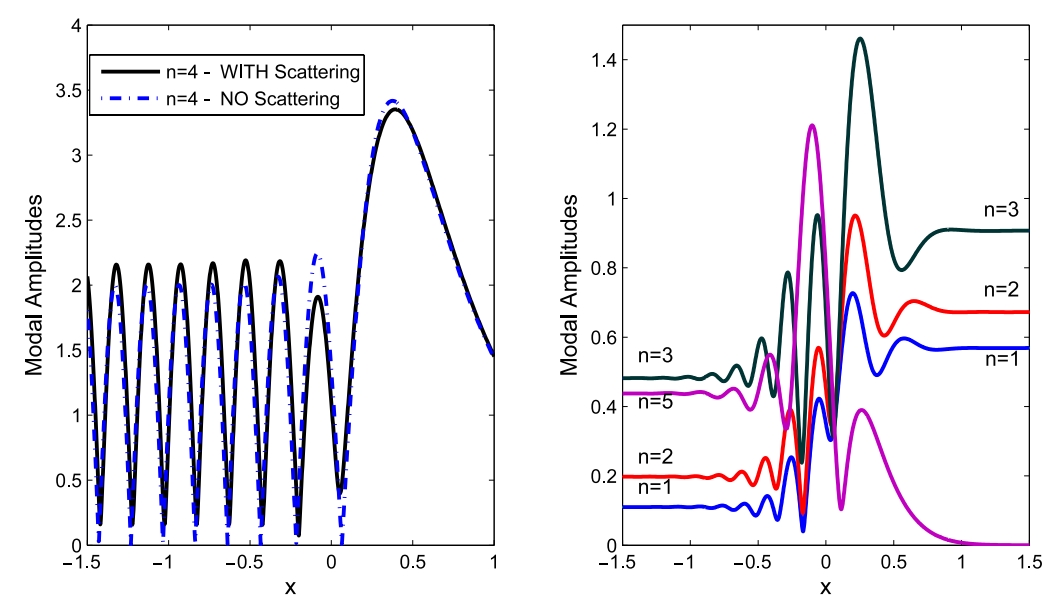

Fig. 3(e). Case C: $\omega=40,(n, m)=(4,14)$ incident, with $\mathcal{F}=-0.4$. The left plot shows the amplitude for the incident mode obtained from the scattering model (solid line) compared to the expected standing wave from the $\omega \sim 1$ multiple scales theory (dotted line). The right plot shows the scattered amplitudes for the neighbouring modes.
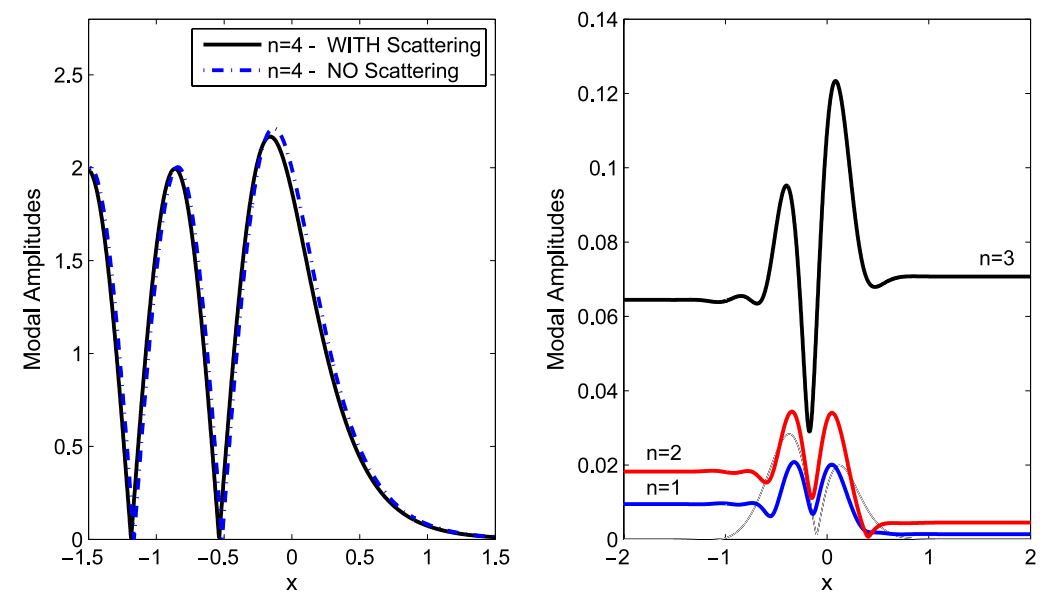

Fig. 3(f). Case C: $\omega=46,(n, m)=(4,14)$ incident-no flow comparison. The left plot shows the amplitude for the incident mode obtained from the scattering model (solid line) compared to the expected standing wave from the $\omega \sim 1$ multiple scales theory (dotted line). The right plot shows the scattered amplitudes for the neighbouring modes.

are much less significant for this case. Such negligible scattering without mean flow can be explained due to the fact that the varying wavenumber coefficient in the $z$-direction $n \pi / h(x) \ll \omega$ for the incident and neighbouring modes and thus $\alpha_{n}^{\prime}(x)=\beta_{n}^{\prime}(x) \ll \omega$ and the driving force of the geometry-induced scattering is lessened. Indeed, one can estimate that here $n \pi \sim \epsilon^{-1}$ and so, looking at term 3 in Section 4, we find

$$
\epsilon\left\{\frac{2 \mathrm{i} \omega}{C_{0}^{2}}\left(\mathbf{V}_{\perp} \cdot \nabla_{\perp} \phi\right)\right\} \sim \epsilon \omega n \pi U_{0} \sim \epsilon^{-2}
$$

which is in this case comparable to terms 1 and 2 in Section 4 , of magnitude $\omega^{2}\left|X-X_{t}\right|$, in the inner region. This observation agrees with the scattering effects seen previously [8] where the nondimensional frequency is much higher than the radial mode number (as the azimuthal wavenumber is large) and hence scattering is only observed in the mean flow case.

\section{Conclusions}

The ability to understand and capture modal interactions at high frequency using asymptotic techniques should not only provide useful benchmarks for numerical schemes, but also a more widespread adoption of the multiple-scales approach. The multiple-scales analysis presented here for high Helmholtz numbers has led to the identification of two mechanisms that allow a mode undergoing cut-on cut-off transition to interact with any crosswise mean flow and scatter energy into neighbouring cut-on modes. Conversely it is also possible for other propagating modes present to interact and alter a standing wave within the duct. While high-frequency scattering can be induced purely by geometry variations, our analysis and results demonstrate that flow-induced scattering when a mean flow is present appears to be significant at much lower 
Table 1

Case B: Incident modal amplitudes $\mathcal{A}_{n}$, and the absolute values of both reflected modal amplitudes $\mathcal{R}_{n}$ and transmitted modal amplitudes $\mathcal{T}_{n}$ (to 2 decimal places) for the two mean flow scenarios $\mathcal{F}= \pm 0.4$

\begin{tabular}{rlllrlll}
\hline \multicolumn{1}{r}{$n$} & $\mathcal{A}_{n}$ & $\left|\mathcal{R}_{n}\right| U_{0}>0$ & $\left|\mathcal{T}_{n}\right| U_{0}>0$ & \multicolumn{1}{c}{$n$} & $\mathcal{A}_{n}$ & $\left|\mathcal{R}_{n}\right| U_{0}<0$ & $\left|\mathcal{T}_{n}\right| U_{0}<0$ \\
\hline 8 & 0 & 0.01 & 0.00 & 8 & 0 & 0.00 & 0.00 \\
9 & 0 & 0.01 & 0.00 & 9 & 0 & 0.00 & 0.00 \\
10 & 0 & 0.03 & 0.00 & 10 & 0 & 0.00 & 0.02 \\
11 & 0 & 0.07 & 0.01 & 11 & 0 & 0.00 & 0.07 \\
12 & 0 & 0.17 & 0.05 & 12 & 0 & 0.02 & 0.15 \\
13 & 0 & 0.48 & 0.26 & 13 & 0 & 0.11 & 0.26 \\
14 & 1 & 1.55 & Cut-off & 14 & 1 & 0.53 & Cut-off \\
15 & 0 & Cut-off & Cut-off & 15 & 0 & Cut-off & Cut-off \\
\hline
\end{tabular}

Table 2

Case C: Incident modal amplitudes $\mathcal{A}_{n}$, and the absolute values of both reflected modal amplitudes $\mathcal{R}_{n}$ and transmitted modal amplitudes $\mathcal{T}_{n}$ (to 2 decimal places) for the two mean flow scenarios $\mathcal{F}= \pm 0.4$.

\begin{tabular}{llllllll}
\hline$n$ & $\mathcal{A}_{n}$ & $\left|\mathcal{R}_{n}\right| U_{0}>0$ & $\left|\mathcal{T}_{n}\right| U_{0}>0$ & $n$ & $\mathcal{A}_{n}$ & $\left|\mathcal{R}_{n}\right| U_{0}<0$ & $\left|\mathcal{T}_{n}\right| U_{0}<0$ \\
\hline 1 & 0 & 0.04 & 0.01 & 1 & 0 & 0.11 & 0.57 \\
2 & 0 & 0.11 & 0.03 & 2 & 0 & 0.20 & 0.67 \\
3 & 0 & 0.40 & 0.49 & 3 & 0 & 0.48 & 0.91 \\
4 & 1 & 0.44 & Cut-off & 4 & 1 & 1.16 & Cut-off \\
5 & 0 & 0.19 & Cut-off & 5 & 0 & 0.44 & Cut-off \\
\hline
\end{tabular}

Helmholtz numbers. Our initial numerical results also suggest that scattering allows energy to propagate beyond the cut-on cut-off transition point contrary to the prediction of standard multiple-scales theory for duct modes. More details on the rational asymptotic structure for the flow-induced scattering mechanism will be provided in the forthcoming Ph.D. thesis of Alex Smith.

The degree of modal interaction for the flow-induced mechanism is determined principally by the scattering coefficients $\mathcal{C}_{k, n}$, which are most significant between the nearest eigenvalue neighbours where $|n-k|$ is small. However, the direction of the mean flow through the geometry variation also appears to dictate both the strength of the scattering into neighbouring modes as well as the strength of the reflected counterpart of the incident mode. Indeed, for a mean flow that accelerates and constricts through a narrowing, over-reflection can occur where the reflection coefficient of the incident mode can be significantly larger than one. This along with the other evidence presented clearly highlights that the interaction between the acoustic field and crosswise mean flow leads to an exchange of energy between the two. However, it is important to note that due to the linear assumption of the acoustic field being infinitesimally small, the coupling results in a negligible impact on the mean flow and indeed the mean flow, in turn, represents a practically infinite source of energy for the acoustic field. The impact of the flow-induced scattering mechanism appears especially significant in three-dimensional cases where, in case $\mathrm{C}$ presented, we demonstrate an amplified transmitted acoustic field generated by an incident mode that undergoes cut-on cut-off transition within a duct; this result is at complete odds to the trapped standing wave that would be predicted by standard multiple-scales theory.

While the initial results presented here are for a duct of rectangular cross section, we note that our analysis applies equally and straightforwardly to ducts of hollow-circular or annular cross section more akin to aeroengine geometries. The minor added complication for a hollow cylindrical duct, for instance, is that the calculation of both scattering coefficients, $\mathcal{C}_{k, n}$ and $g_{k, n}$, requires integration over the duct cross section of $r J_{m}^{\prime}\left(\beta_{n}(X) r\right) J_{m}\left(\beta_{k}(X) r\right)$ and $r^{2} J_{m}^{\prime \prime}\left(\beta_{n}(X) r\right) J_{m}\left(\beta_{k}(X) r\right)$ respectively for a given azimuthal wavenumber $m$. For annular ducts the calculation of scattering coefficients is complicated further to a degree as $\mathcal{C}_{k, n}$ and $g_{k, n}$ will be a function of the ratio between the inner and outer radii, which is likely to be slowly-varying along the duct. However, such scattering calculations only require a look-up table and should remain extremely fast and efficient compared to alternative approaches.

The theory and results presented here provide some encouraging agreement with the finite-element simulations shown in the previous comparison paper of Ovenden et al. [8] and a more quantitative comparison is planned for the future. In fact, we note that reflection coefficients for the incident mode obtained in cases 4 and 6 of that paper were of the order of 0.6 and lower. Given the directional sense of mean flow, so that it is diverging rather than converging at the transition point in those cases, this agrees very well with the predictions of our theory. On a final note, due to the fact that over-reflection is predicted in some cases, the resulting higher amplitude standing waves generated may have some important consequences in managing the structural integrity of aeroengines. This remains true even in lined ducts where partial reflections are already predicted by the $\omega \sim 1$ theory [12]. We hope to report more analysis on such issues in a future publication. 


\section{Acknowledgements}

The authors are grateful to the Engineering and Physical Sciences Research Council for funding Alex Smith's Ph.D. research. We would also like to thank Sjoerd Rienstra, Walt Eversman and Nigel Peake for their encouragement and interest in this problem and also to the referees for their interest and constructive comments. Finally, we are indeed grateful to one referee for highlighting a useful scaling for $U_{0} \ll 1$ in the inner scaling region for high frequencies.

\section{References}

[1] A.H. Nayfeh, D.P. Telionis, Acoustic propagation in ducts of varying cross sections, Journal of the Acoustical Society of America 54 (1973) 1654-1661.

[2] S.W. Rienstra, Sound transmission in slowly varying circular and annular ducts with flow, Journal of Fluid Mechanics 380 (1999) $279-296$.

[3] A.J. Cooper, N. Peake, Propagation of unsteady disturbances in a slowly varying duct with mean swirling flow, Journal of Fluid Mechanics 445 (2001) 207-234.

[4] S.W. Rienstra, Sound propagation in slowly varying lined flow ducts of arbitrary cross section, Journal of Fluid Mechanics 495 (2003) 157-173.

[5] E.J. Brambley, N. Peake, Sound transmission in strongly curved slowly varying cylindrical ducts with flow, Journal of Fluid Mechanics 596 (2008) 387-412.

[6] I. Danda-Roy, W. Eversman, Improved finite element modeling of the turbofan engine inlet radiation problem, ASME. Journal of Vibration and Acoustics 117 (1995) 109-115.

[7] S.W. Rienstra, W. Eversman, A numerical comparison between the multiple-scales and finite-element solution for sound propagation in lined flow ducts, Journal of Fluid Mechanics 437 (2001) 367-384.

[8] N.C. Ovenden, W. Eversman, S.W. Rienstra, Cut-on cut-off transition in flow ducts: comparing multiple-scales and finite-element solutions, in: Paper AIAA 2004-2945 of the 10th AIAA/CEAS Aeroacoustics Conference, Manchester, UK, 10-12 May.

[9] S.W. Rienstra, Cut-on cut-off transition of sound in slowly varying flow ducts, Aerotechnica - Missili e Spazio, in: L. Morino and N. Peake (Eds.) (special issue in memory of Prof. D.G. Crighton), vol. 79, 2000, 93-97.

[10] X.D. Li, C. Schemel, U. Michel, F. Thiele, On the azimuthal mode propagation in axisymmetric duct flows, in: Paper 2002-2521 of the 8th AIAA/CEAS Aeroacoustics Conference in Breckenridge, CO, USA, 17-19 June.

[11] N.C. Ovenden, A uniformly valid multiple scales solution for cut-on cut-off transition of sound in flow ducts, Journal of Sound and Vibration 286 (2005) 403-416.

[12] N.C. Ovenden, Near cut-on/cut-off transitions in lined ducts with flow, in: Paper AIAA 2002-2445 of the 8th AIAA/CEAS Aeroacoustics Conference in Breckenridge, CO, 17-19 June.

[13] M.S. Howe, Acoustics of Fluid Structure Interactions, Cambridge University Press, Cambridge, 1998, pp. 27-33.

[14] S.W. Rienstra, Acoustic scattering at a hard-soft lining transition in a flow duct, Journal of Engineering Mathematics 59 (2007) 451-475.

[15] E.J. Brambley, Low-frequency acoustic reflection at a hard-soft lining transition in a cylindrical duct with uniform flow, Journal of Engineering Mathematics 65 (2009) 345-354.

[16] M.K. Myers, An exact energy corollary for homentropic flow, Journal of Sound and Vibration 109 (1986) 277-284. 